\title{
Efficient Movement Compensation and Detection Algorithm using Blob Detection and Modified Kalman Filter
}

\author{
Sridevi N, M Meenakshi
}

\begin{abstract}
In various real time applications such as security and surveillance etc., detection of movement from video sequence is commonly used. In such applications, time required to detect the movement and its accuracy is very crucial. In this paper, an efficient motion compensation and detection algorithm using Blob detection and modified Kalman filter techniques is proposed. The method is mainly based on Kalman filtering technique which is modified to compensate and detect the unwanted movement caused by the camera. Also the shadow effect caused by the variation in the intensity of light and object is removed using thresholding technique. Accuracy of movement detection is improved by implementing the blob detection method. The experimental results obtained from the developed algorithm is compared with few methods existing in the literature for validation.
\end{abstract}

Keywords: Motion estimation, Motion detection, Motion compensation, Kalman Filter, Thresholding, Three Step Search, Blob detection.

\section{INTRODUCTION}

Literature reveals that the tracking of movement from the video sequence is easier when the camera is static compared with dynamic environment like camera movement [1]. This happen when the camera module is mounted on some moving platform which introduces some adverse effect on the captured video such as blurns and haziness etc. In such cases it is difficult to detect moving objects using conventional methods. Since it requires highly complex mathematical expression and hence the computational time requirement which makes it unsuitable for real time applications. Therefore this necessitates improvised algorithms to estimate, detect the movement and compensate the unwanted noises present in the video sequences. To address the issues discussed above many researchers have proposed different methods some of them are discussed below. Adaptive window size selection based motion estimation algorithm is presented in paper [2]. In this paper the $1 \mathrm{D}$ matching to overcome the original $2 \mathrm{D}$ block matching which reduces the encoding time bi-region similarity detection (BSD) algorithm is used. By implementing the algorithm the authors of the paper archived negligible PSNR loss,

Revised Manuscript Received on June 15, 2020.

* Correspondence Author

Sridevi N*, Department of EIE, Dr. Ambedkar Institute of Technology, Bangalore, Karnataka, India. Affiliated to VTU, Belagavi, Karnataka Email: sridevee@gmail.com.

M Meenakshi, Department of EIE, Dr. Ambedkar Institute of Technology, Bangalore, Karnataka, India. Email: meenakshi_mbhat@yahoo.com.

(C) The Authors. Published by Blue Eyes Intelligence Engineering and Sciences Publication (BEIESP). This is an open access article under the CC BY-NC-ND license (http://creativecommons.org/licenses/by-nc-nd/4.0/)
Increments in the bit rate and time reduction is greater than $60 \%$. Real time motion estimation using real time camera through robust feature is presented in [3]. Here the parameters required for the camera motion model is calculated using the motion vectors from the set of selected points. However only the motion model that had the largest number of inliers was returned as a result with camera motion estimation capability. Authors of the paper [4] proposed an algorithm to estimate the motion based on inter-frame differences detection. Here the results demonstrate the algorithm is suitable for detecting under complex background the direction of small moving object with better real time performance. However the limitation in this case is that the algorithm is designed only for rectangular shaped object detection. Next the motion estimation using edge matching is presented in [5]. Where to create the edges authors of the paper used canny edge detector. Here in this paper the improvement in the motion estimation accuracy in terms of PSNR is given. The limitation of this paper is that the algorithm gives better estimation accuracy only when the video contains small number of objects and it fails for larger number of objects. To identify and appraise movement in a video coding framework an Error Detection and Correction Architecture (EDCA) is planned dependent on the Residue-and-Quotient (RQ) code which is introduced in paper [6]. The proposed plan had the option to distinguish various piece blunders and successfully recouped the information with decreased territory. Creators in paper [7] introduced Modified-Octagon Based Search calculation (MOCTBS) to evaluate movement. MOCTBS utilizes little octagon example to pass judgment if the MBD (Minimal Block Distortion) point situated in the hover of one pixel around the underlying hunt community. To accelerate the movement estimation, zero-square discovery strategy is utilized. The MOCTBS speeded up the movement estimation process extraordinarily under a similar encoding productivity. The confinement of this strategy is MOCTBS may likewise be caught in neighborhood minima. Next the movement vector estimation from observation video edges to identify movement is introduced in [8]. Movement is recognized with an edge area assurance approach which makes discovery quicker. Here optical stream with Horn-Schunck calculation is utilized for movement estimation. The calculation needs less computational expense and it is liberated from natural and moving camera condition.

Published By:

Blue Eyes Intelligence Engineering 
Paper [9] utilizes iterative movement estimation and mistake assessment technique for effective impediment location. This technique is proposed with the end goal of PC vision regions that need precise impediment discovery. This technique recognizes the impeded districts. Creators of the paper [10] utilized movement estimation and revision unit. Movement estimation unit depends on square coordinating to appraise worldwide movement parameters expected to make up for the jitter and twist the picture to yield a settled grouping. The impediment in this technique is that the amended video picture is marginally obscured and the image goals is somewhat decreased.

From the above literature surveys, it is observed that the existing algorithms can't be able to detect and compensate the movement properly. As a result, the detection accuracies are less for those cases. Moreover, if the camera module is in motion then the detection accuracy reduced drastically. To overcome from the above discussed issues, in this paper conventional kalman filter equation is modified to improve the accuracy of detection in a dynamic environment.

\section{PROPOSED ARCHITECTURE}

The proposed motion estimation and detection algorithm is given in figure 1.

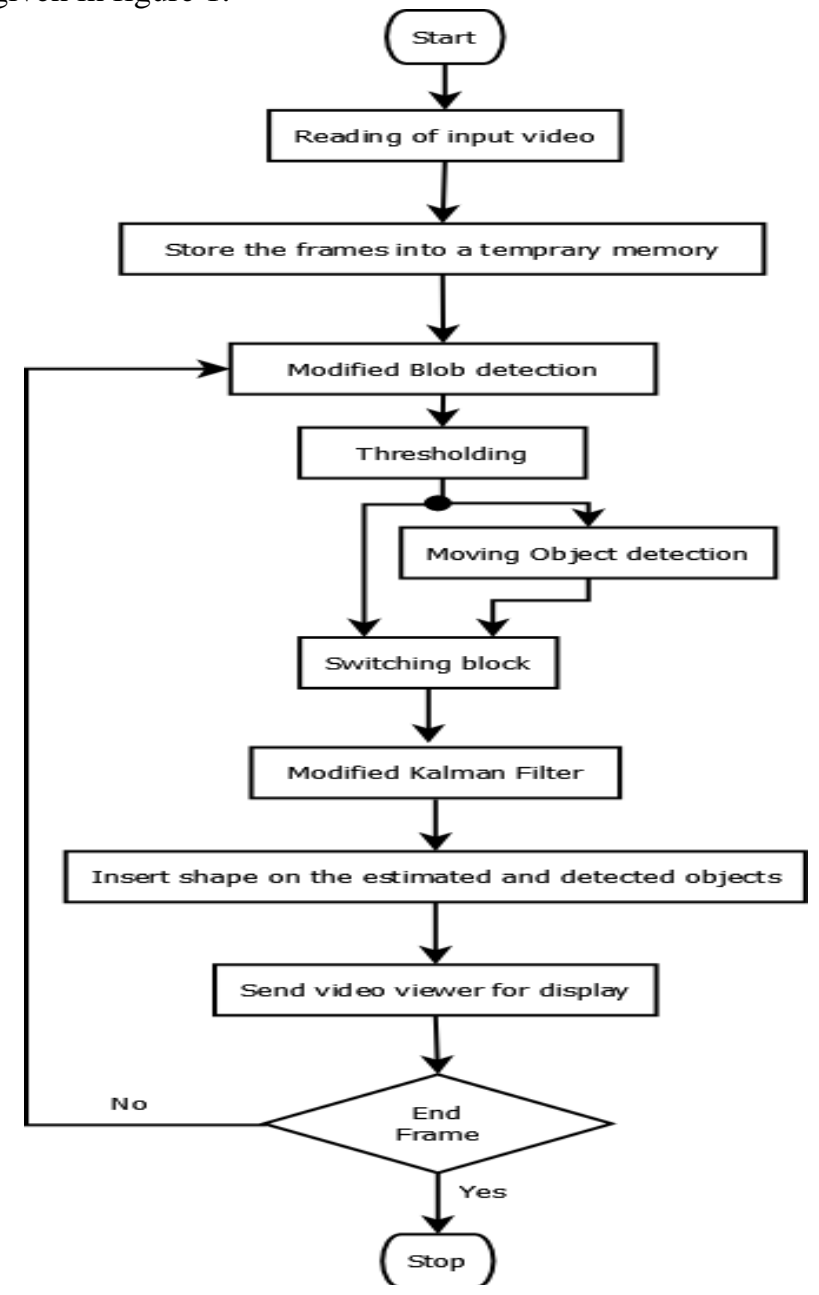

Fig. 1. Flow diagram of motion estimation and detection algorithm

In this case, first the input video is read which is then converted into a finite number of frames depending upon video specifications such as frame rate, frame size etc. The corresponding blobs are detected from those frames using Modified Blob Detection technique which consists of some amount of noises. Those noises are removed by Thresholding block from which the moving object is detected by Moving Object Detection block. The output of the thresholding and Moving Object Detection is connected to the Switching block which switches the signal for proper manner to help the Modified Kalman Filter block to perform its proper operation. This signal is used by the Modified Kalman Filter to estimate, predict and correct the motion resulting almost accurate motion detection and compensation. Now insert the bounding box to the detected objects and display it using video viewer. Repeat the total operation in circular manner until all the frames are processed. Each block in the flow diagram is explained in detail below.

\section{A. Reading of the input video}

Normally videos are stored in the format of mp4, avi etc. To read the stored video signal, video recorder present in Image processing toolbox of MATLAB tool[11] is used. This reader is able to read most of the available video format exist till today.

\section{B. Converting the video into number of frames}

The video stream read by MATLAB video reader tool box is not suitable for processing. For proper processing, it is required to convert the video stream into a finite number of frames [11]. At the time of conversion, the frame rate must be same as it is in the video format. Otherwise different types of issues occur in the design which leads to wrong prediction and detection.

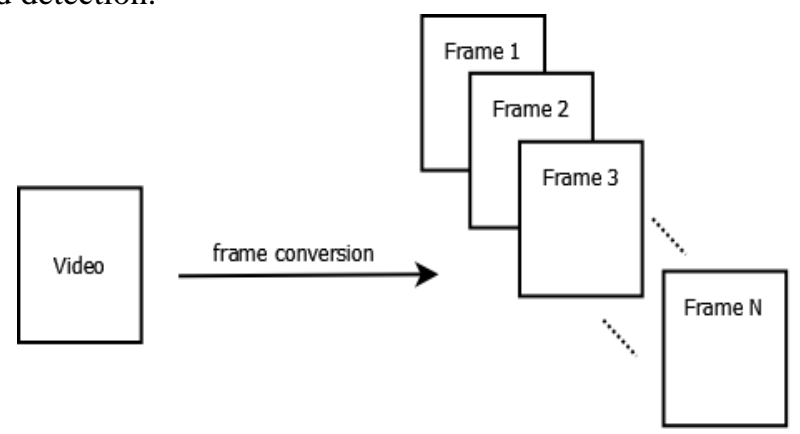

Fig. 2.Different frames of a video

\section{Blob detection}

In digital image processing Blob analysis is given by detecting regions that is having different properties from its adjacent regions. Here in this paper statistical blob technique is used [21]. An example of blob detection is given in figure 3.

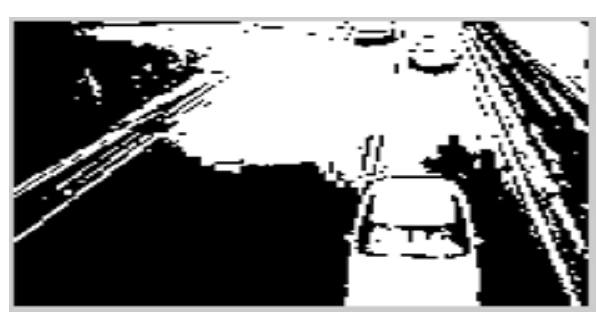

Fig. 3.Blob Analysis

\section{Published By:}

Blue Eyes Intelligence Engineering \& Sciences Publication 
In this method, the different features of geometric parameters such as area, boarder line, center etc, present in the input image is represented by creating symbols from the statics of blobs. However the output of the morphological operation is used to extract blobs. In this application the normal statistical blob [22] is not suitable. Hence it is necessary to modify the existing statistical [23] blob method. In this paper the blob detection method proposed in the paper [26] is used.

\section{Thresholding}

To reduce the false movement detection due to the light intensity variations, thresholding operation is used. Normally, any thresholding operation for image processing is mainly divided into two types as soft thresholding and hard thresholding [12]. In this case soft thresholding is used. To reduce noise easily and effectively, soft thresholding is used where the coefficient is zero when the absolute values are less than a threshold, otherwise, the coefficients value is modified and is given in equation (1).The result of the thresholding is assigned to the value of the sign function which multiplies the subtraction value between a coefficient and threshold T. If the coefficient is equal 0 , greater than zero, or less than zero, then the sign function returns $0,1,-1$ respectively.

$f_{S}=\left\{\begin{array}{cc}\operatorname{sgn}(\mathrm{C}(\mathrm{n}))(\mathrm{C}(\mathrm{n})-\mathrm{T}) ; \text { for }|\mathrm{C}(\mathrm{n})| \geq \mathrm{T} \\ 0 ; & \text { Otherwise }\end{array}\right.$

\section{E. Moving Object Detection}

The displacement of any object in the video sequence is known as moving object. This is mainly determined by observing the difference between two or more consequent frames. Three step search (TSS) $[13,14]$ is used in this paper to detect the moving object present in the frame. This method is based upon fast search technique which is having capability to reduce the candidate blocks effectively without reducing the search area. The algorithm is used to perform TSS is given in Figure 4. To perform moving object detection, three step search first set zero to the motion vector and try to find the best match value of the block in the current frame and reference frame. If and only if the best match value is lesser than a predefined threshold then only the minimum value of the best match set is considered as the centroid of motion in the reference frame from a set of current match frames. Using this best match, the centroid list is updated. If the step size is larger than ' 1 ', then half of the step size is considered and then the centroid list is updated else motion vector is returned. The sum of absolute difference (SAD) [15] is used for matching where the SAD values between two blocks are considered.

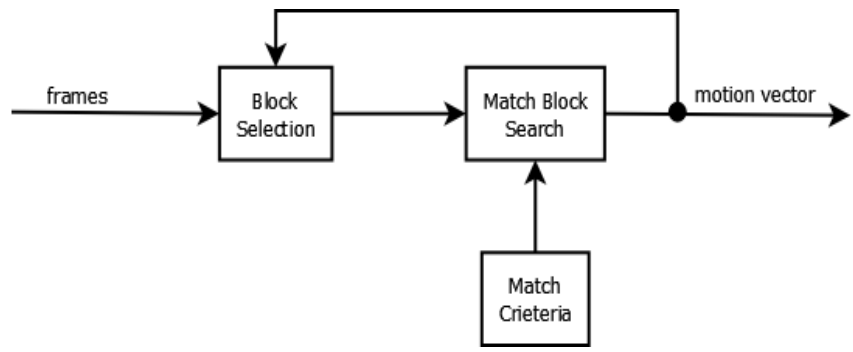

Fig. 4.Three step search algorithm
Let us consider for $\mathrm{n} \times \mathrm{n}$ pixel blocks, the matching criterion $\mathrm{M}(\mathrm{p}, \mathrm{q})$ is defined by the following equation where $(p, q)$ define the displacement. The block with the smallest $\mathrm{SAD}$ value is selected as the best match block, and the motion vector is then calculated accordingly.

$M(p, q)=\sum_{i=1}^{n} \sum_{j=1}^{n}\left[I_{c}(i, j)-I_{r}(i+p, j+q)\right]$

\section{F. Switching Block}

The modified Kalman filter needs some set of predefined detected frames from which the initial motion vectors are fed to the Modified Kalman filter. Those vectors are needed at initial conditions only. After some time the Modified Kalman filter can predict it by itself. So, it is necessary to disconnect the Moving Object Detection block output after some initial frames which is done by the frame switching block.This block switches the signal after 15 frames.

\section{G. Modified Kalman Filter}

The Kalman filter is used to predict and correct the states present in many linear mathematical environment [16]. Normally due to the most accurate prediction and correction capabilities in both theoretical and practical environments, the kalman filter is used more widely than any other similar kind of filter. The prediction equation and update equation is used in Kalman filtering is given in Eq. (3) and Eq. (4)

$X(n)=F \cdot X(n-1)+V_{q}(n-1)$

$Y(n)=H \cdot X(n)+V_{p}(n)$

Where, $\mathrm{X}(\mathrm{n})$ - Estimated state variable

$\mathrm{Y}(\mathrm{n})$ - Measurement variable

$\mathrm{H}$ - Measurement matrix

$\mathrm{V}_{\mathrm{q}}(\mathrm{n})$ - System noise

$\mathrm{V}_{\mathrm{p}}(\mathrm{n})$ - Measured noise

Now, the basic equations of Kalman filter [17] for image with linear environment is given in equation (5) to equation (12) below

$$
\begin{aligned}
X_{0} & =X, i=0 \\
X_{i} & =X+\sqrt{(N+\lambda) P_{i}}, i=1,2, \ldots, N \\
X_{i} & =X+\sqrt{(N+\lambda) P_{(i-N)}}, i=N+1, N+2, \ldots, 2 N \\
w_{0}^{m} & =\frac{\lambda}{(N+\lambda)} \\
w_{0}^{c} & =\frac{\lambda}{(N+\lambda)+\left(3-\alpha^{2}\right)} \\
w_{i}^{m} & =w_{0}^{c}=\frac{1}{2(N+\lambda)}, i=1,2, \ldots, 2 N \\
w^{m} & =\left[w_{0}^{m}, \ldots, w_{2 N}^{m}\right]^{T} \\
w & =\left(I-\left[w^{m} \ldots w^{m}\right]\right) * \operatorname{diag}\left(w_{0}^{c} \ldots w_{2 N}^{c}\right) *(I- \\
{\left[w^{m}\right.} & \left.\left.\ldots w^{m}\right]\right)
\end{aligned}
$$

Where, $\lambda=3 \alpha^{2}-N$ and $\alpha$ controls the range of samples.

The above equation of Kalman filter is mainly based on linear errors which are not suitable for nonlinear case.

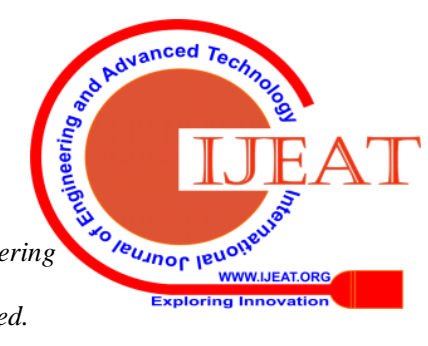




\section{Efficient Movement Compensation and Detection Algorithm using Blob Detection and Modified Kalman \\ Filter}

To overcome from this problem, unscented transform [16] is used in Kalman filter prediction and update equations which is known as Unscented Kalman filter [17, 27]. This is widely used in real time scenario. The prediction is represented in nonlinear variation scenario as

$$
\begin{aligned}
& X(n+1)=f\{X(n)\} \\
& Y(n)=h\{X(n)\}
\end{aligned}
$$

The existing equation for kalman filter with camera motion [28] is modified to get the modified Kalman filtering equations. To reduce the processing time, the number of the frames used for detection and compensations are reduced to some adjacent frames only. This increases the detection accuracy by considering local detections. But the disadvantages of this local detection is the increment of the false detection which is then minimized by using Blob detection along with the modified Kalman filter. The mathematical equations of modified Kalman filter for nonlinear environment is then defined through Eq (5) to Eq (12) as

$$
\begin{aligned}
& \hat{X}(n)=f\{n-1, X(n-1)\} \\
& \hat{X}\left(n \mid Y_{n-1}\right)=\hat{X}(n) * w^{m} \\
& P(n, n-1)=\left\{\hat{X}_{n} * W\left(\hat{X}_{n}\right)^{T}\right\}+V_{q}(n-1) \\
& X(n)=[\hat{X}(n \mid Y(n-1)) \ldots \hat{X}(n \mid(n-1))]+ \\
& \sqrt{3} \alpha[\sqrt{P(n-1)}-\sqrt{P(n)}] \\
& Y(n)=h\{n, X(n)\} \\
& K(n)=\frac{X(n) * w * T^{T}(n)}{\left\{Y(n) * w * Y^{T}(n)\right\}+V_{-} p(n)} \\
& \hat{X}\{n \mid Y(n)\}=\hat{X}\{n \mid Y(n-1)\}+K(n)[Y(n)-\{Y(n) * \\
& \left.w^{m}\right\} \\
& P(n)=P(n, n-1)+K(n)\left[\left\{Y(n) * w * Y^{T}(n)\right\}+\right. \\
& \left.V_{p}(n)\right] K^{T}(n)
\end{aligned}
$$

Where, $\hat{X}(n \mid Y(n))$ is the current estimation of state variable $X(n)$ under the observation of $Y(n)$.

Now let us consider in real time scenario, $\mathrm{M}$ moving object exists. So, the measurement vector

$$
\begin{aligned}
& Y=\left[x_{1}, y_{1}, x_{2}, y_{2}, \ldots \ldots, x_{M}, y_{M}\right]^{T} \\
& \text { Where, }\left\{\left(\mathrm{x}_{\mathrm{i}}, \mathrm{y}_{\mathrm{i}}\right) \mid \mathrm{i}=1,2, \ldots \ldots, \mathrm{M}\right\}
\end{aligned}
$$

By considering the velocity and acceleration of the moving object, the equation of variable ' $\mathrm{X}$ ' is

$$
\begin{aligned}
& X= \\
& {\left[x_{1}, v_{x 1}, a_{x 1}, y_{1}, v_{y 1}, a_{y 1}, \ldots \ldots, x_{M}, v_{x M}, y_{M}, v_{y M}, a_{y m}\right]^{T}}
\end{aligned}
$$

Where, $\left\{\left(\mathrm{v}_{\mathrm{xi}}, \mathrm{v}_{\mathrm{yi}}\right) \mid \mathrm{i}=1,2, \ldots \ldots, \mathrm{M}\right\}$ - velocity

$$
\left\{\left(a_{x i}, a_{y i}\right) \mid i=1,2, \ldots \ldots, M\right\}-\text { Acceleration }
$$

The moving model for $\mathrm{i}^{\text {th }}$ object then can be defined as $x_{i}(n)=x_{i}(n-1)+v_{x i}(n-1) d t+\frac{1}{2} a_{x i}(n-1) d t^{2}$

$y_{i}(n)=y_{i}(n-1)+v_{y i}(n-1) d t+\frac{1}{2} a_{y i}(n-1) d t^{2}$
The transition matrix is then

$$
F=\left[\begin{array}{ccc}
F_{1} & \cdots & 0 \\
0 & & 0 \\
\vdots & F_{i} & \vdots \\
0 & \cdots & F_{M}
\end{array}\right]
$$

Where,

$$
F_{i}=\left[\begin{array}{cccccc}
1 & d t & \frac{d t^{2}}{2} & 0 & 0 & 0 \\
0 & 1 & d t & 0 & 0 & 0 \\
0 & 0 & 1 & 0 & 0 & 0 \\
0 & 0 & 0 & 1 & d t & \frac{d t^{2}}{2} \\
0 & 0 & 0 & 0 & 1 & d t \\
0 & 0 & 0 & 0 & 0 & 1
\end{array}\right]
$$

In this case of the proposed modified kalman filter, the initial moving object is detected using TSS which is then used to define initial value of ' $\mathrm{F}$ ' and ' $\mathrm{H}$ ' which are then used to define ' $\mathrm{X}$ ' values. This is performed by using Eq (17) to Eq (19). Then the position and velocity of the moving object is predicted using Eq (6) to eq (12). The predicted velocity and acceleration is fed back to the predictionwhich is used for proper object detection. Each object location is updated using Eq (11). This process continues until all frames are finished.

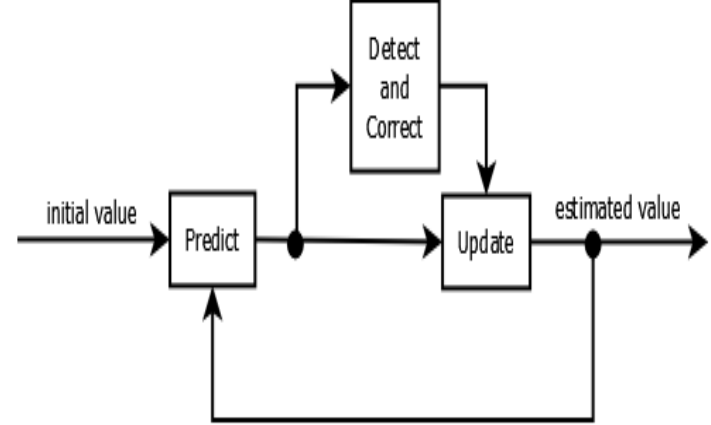

Fig. 5.Proposed Modified Kalman Filter

\section{PERFORMANCE ANALYSIS}

The performance of the designed algorithm is validated by estimating the accuracy of detection and false detection rate from the standard video steams which is discussed in detail below.

\section{A. Operational Parameters}

The accuracy of detection using any detection technique is given by the percentage of ratio of total number of objects detected with the actual number of objects present at a particular time in the video frame and is written mathematically [18] as

$$
\text { Detection Accuracy }=\frac{\text { Number of Detected objects }}{\text { Number of Actual Objects }} \times 100
$$

For acceptable detection accuracy, for many of the frames high percentage of detection accuracy is obtained using eq. (29). However the detection accuracy for different frames is different. As a result, it is needed to calculate the average value of the frames. The equation for average detection accuracy is written as

Published By: 
Detection Rate $=\frac{1}{M} \sum_{i=1}^{M}\left(\right.$ Detection Accuracy $_{i}$

Where, $\mathrm{M}$ is the total number of considered frames

In many cases, due to various algorithmic issues some still objects are also detected as moving object which is technically known as false detection. The mathematical equation of false detection is given by

False Detection $=\frac{\text { Number of Falsely Detected Objects }}{\text { Number of Actual Objects }} \times 100$

The False detection rate is then

False Detection Rate $=\frac{1}{M} \sum_{i=1}^{M}(\text { False Detection })_{i}$

\section{B. Simulation Result}

The new algorithm introduced in this paper is implemented using MATLAB 2013a simulation tool. The algorithm is validated by considering different video scenarios which includes normal traffic, dense traffic and aerial traffic.

Normal Traffic: In this case, standard normal traffic video is considered. In this video sequence the number of cars are less which makes the detection easier and accurate. The simulation results are given in Figure 6 with some different frames.From those detected frames, it can be seen that most of the cases the algorithm detects the moving objects correctly, but in small cases it shows some false detections. This is mainly occurs when any new object introduced or background changes then the algorithm needs some time to predict and detect the motion components correctly.

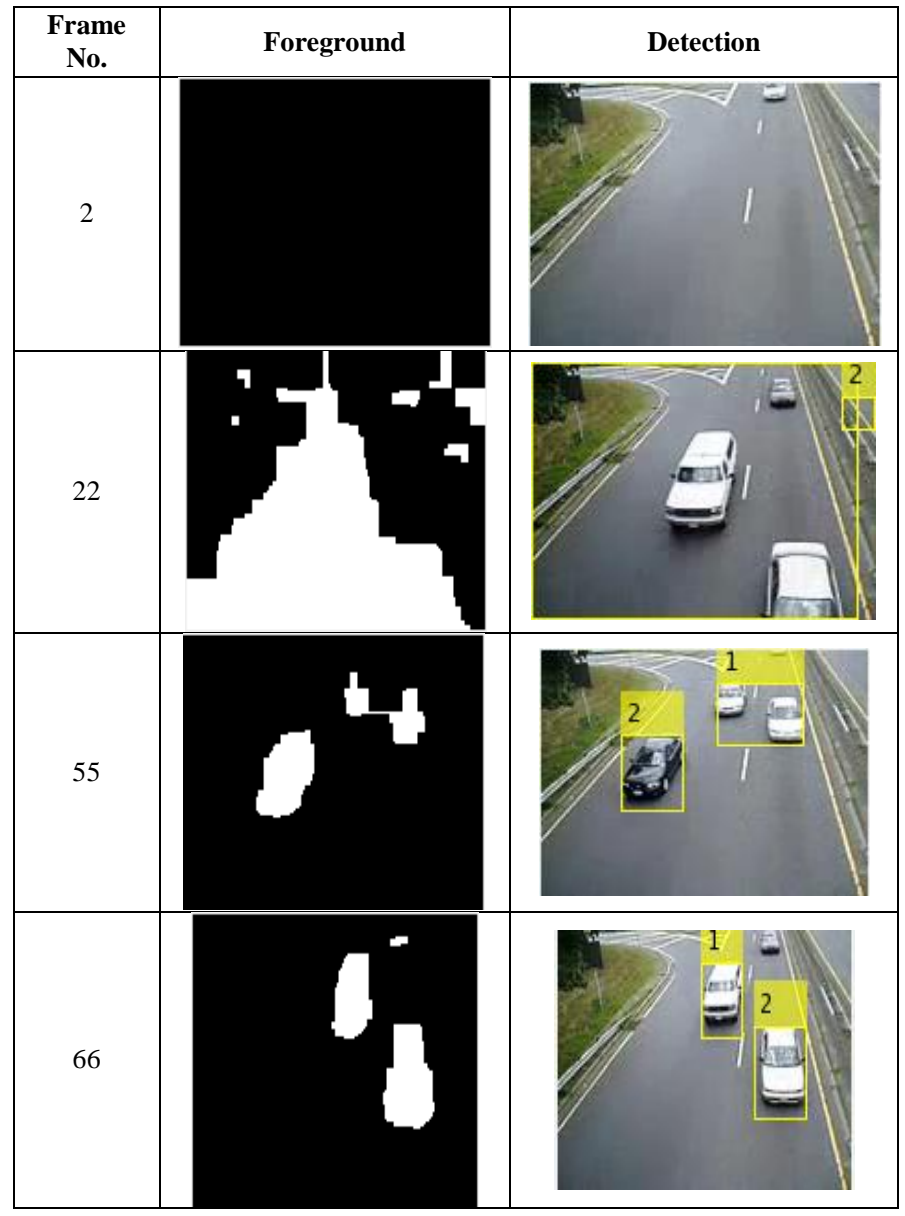

Fig. 6.Simulation Results of normal traffic flow

Table- I: The performance parameters with respect to normal traffic scenario

\begin{tabular}{|c|c|c|c|}
\hline \multirow{2}{*}{$\begin{array}{l}\text { Frame } \\
\text { Number }\end{array}$} & \multicolumn{3}{|c|}{ Moving Object Detection } \\
\hline & Actual Objects & Correctly Detected & $\begin{array}{c}\text { Falsely } \\
\text { Detected }\end{array}$ \\
\hline 2 & 0 & 0 & 0 \\
\hline 17 & 0 & 0 & 0 \\
\hline 22 & 3 & 3 & 0 \\
\hline 30 & 3 & 3 & 0 \\
\hline 35 & 1 & 1 & 1 \\
\hline 42 & 1 & 1 & 0 \\
\hline 55 & 2 & 2 & 0 \\
\hline 65 & 3 & 2 & 0 \\
\hline 66 & 3 & 2 & 0 \\
\hline 69 & 3 & 3 & 0 \\
\hline 70 & 3 & 3 & 0 \\
\hline 87 & 1 & 1 & 0 \\
\hline 108 & 1 & 1 & 0 \\
\hline 110 & 1 & 1 & 0 \\
\hline \multicolumn{2}{|c|}{ True Detection rate } & \multicolumn{2}{|c|}{$97.44 \%$} \\
\hline \multicolumn{2}{|c|}{ False Detection rate } & \multicolumn{2}{|c|}{$3.86 \%$} \\
\hline
\end{tabular}

Dense Traffic: In this case, dense traffic video is considered. In this video sequence the number of cars are more which makes the detection tough and inaccurate. The simulation results are given in Figure 7 for different frames. In this case due to the overlapping of the moving components, the actual detection accuracy is reduced with compared to normal traffic conditions. But in the most of the existing motion detection algorithm can't detect moving objects at high accuracy in dense traffic conditions.

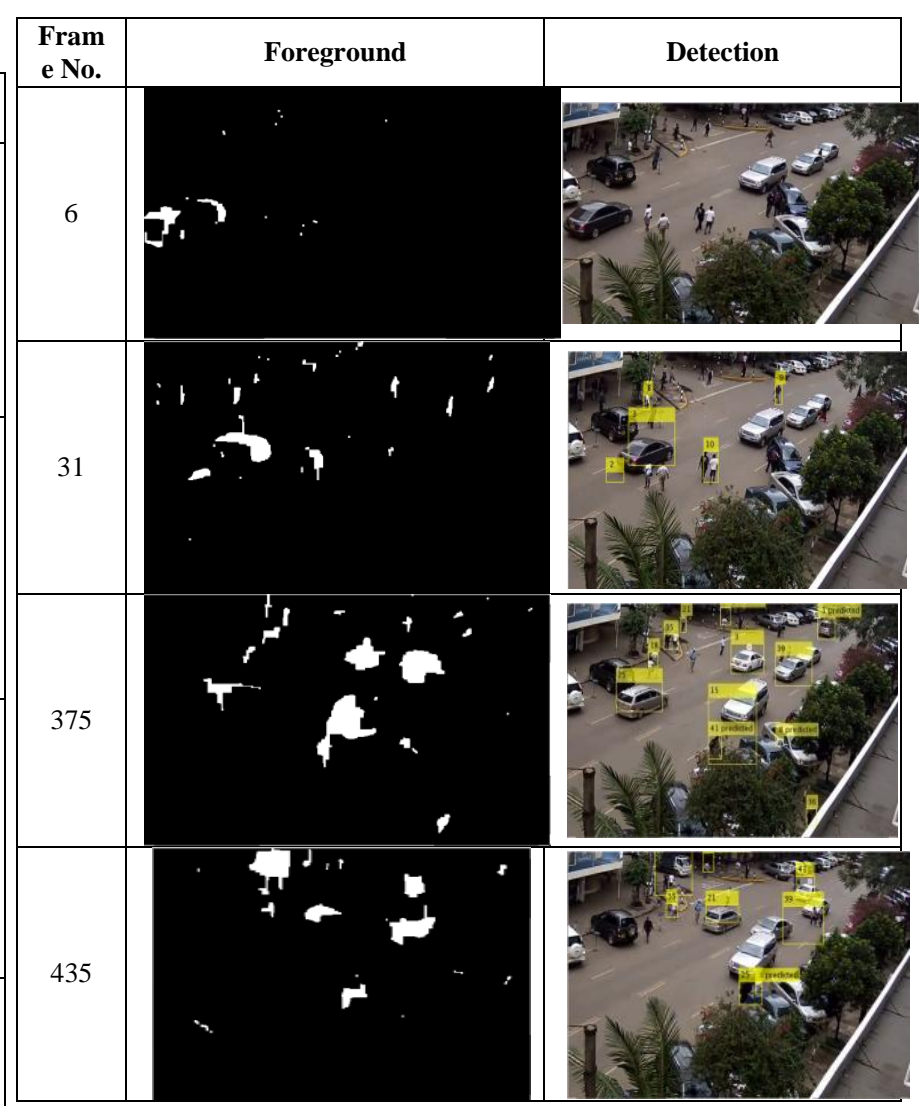

Fig. 7.Simulation Results of dense traffic

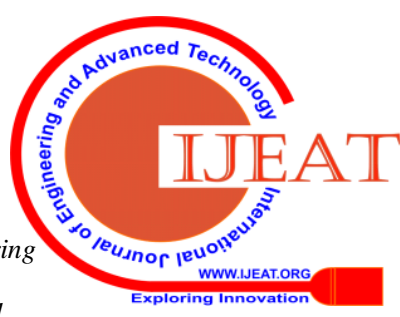


Efficient Movement Compensation and Detection Algorithm using Blob Detection and Modified Kalman Filter

Table- II: Performance parameters for dense traffic.

Table- III: Performance parameters for aerial video

\begin{tabular}{|c|c|c|c|}
\hline \multirow{2}{*}{ Frame Number } & \multicolumn{3}{|c|}{ Moving Object Detection } \\
\cline { 2 - 4 } & Actual Objects & Detected Objects & Falsely Detected \\
\hline 4 & 13 & 2 & 0 \\
\hline 59 & 18 & 12 & 3 \\
\hline 84 & 18 & 14 & 2 \\
\hline 133 & 21 & 16 & 3 \\
\hline 251 & 29 & 21 & 5 \\
\hline 315 & 25 & 19 & 2 \\
\hline \multicolumn{3}{|c|}{ True Detection rate } & $67.74 \%$ \\
\hline \multicolumn{3}{|c}{ False Detection rate } \\
\hline
\end{tabular}

Aerial Image: The aerial videos are captured by a camera mounted on a drone is consider for this case. The foreground and detected objects are given in figure 4 . In the case of the accurate detection when the camera is in motion, special camera with higher frame rates than normal camera is necessary. This will allow the algorithm to create the background model effectively which is helpful for proper detection and estimation. But such videos are not available easily. So, for demo purpose normal video with motion in camera is considered where the above mentioned criterion is not fulfilled. As a result, the detection accuracy is very low in this case.

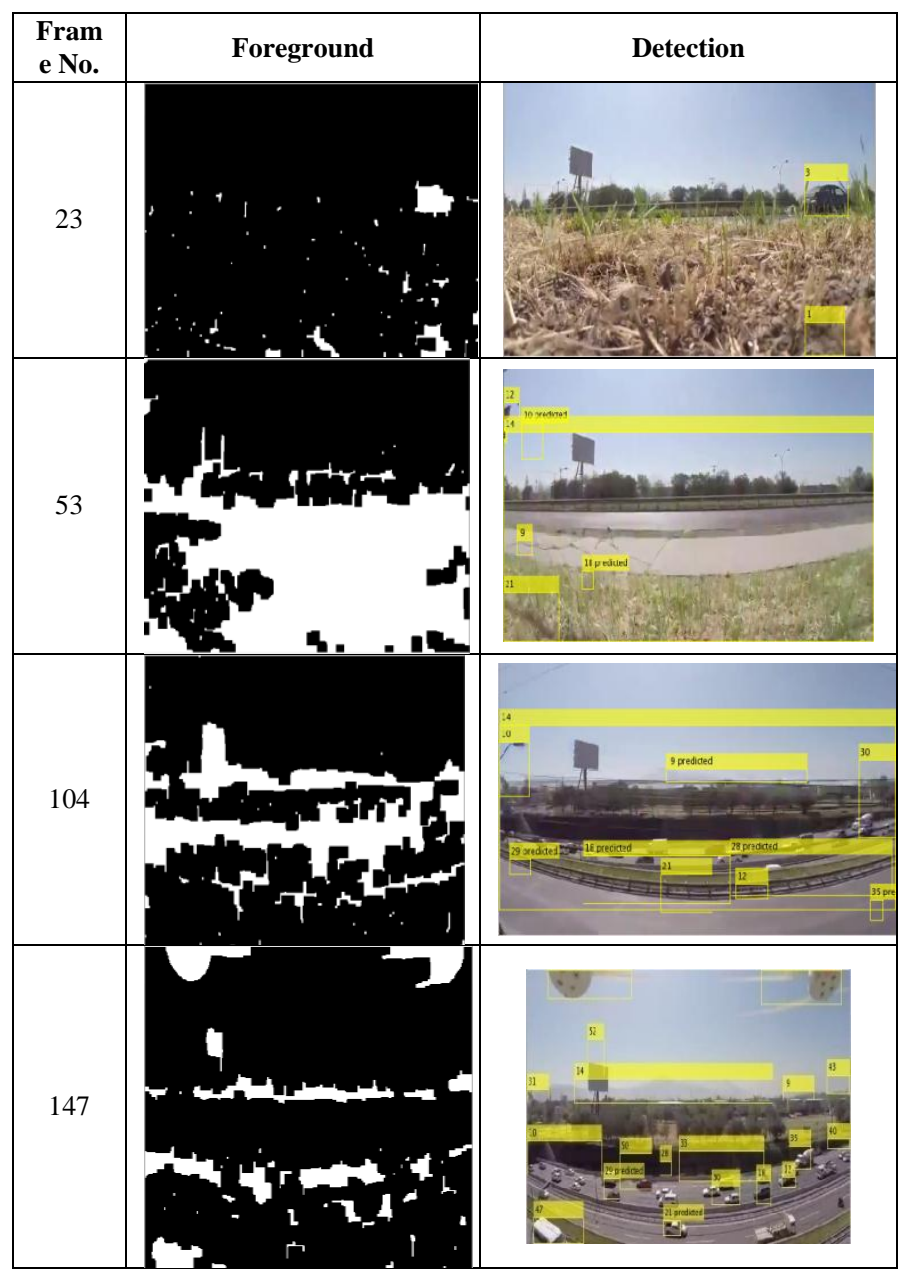

Fig. 8.Simulation Results of Aerial Video

\begin{tabular}{|c|c|c|c|}
\hline \multirow{2}{*}{ Frame Number } & \multicolumn{3}{|c|}{ Moving Object Detection } \\
\cline { 2 - 4 } & Actual Objects & Detected Objects & Falsely Detected \\
\hline 4 & 1 & 1 & 1 \\
\hline Ion & 8 & 4 & 5 \\
\hline 56 & 12 & 8 & 6 \\
\hline 132 & 21 & 15 & 5 \\
\hline 209 & 25 & 16 & 8 \\
\hline 402 & 28 & 17 & 4 \\
\hline \multicolumn{3}{|c|}{ True Detection rate } & $64.21 \%$ \\
\hline \multicolumn{3}{|c}{ False Detection rate } \\
\hline
\end{tabular}

\section{COMPARISONS WITH EXISTING TECHNIQUES}

The results obtained from proposed movement compensation and detection technique is compared with some of the existing methods in terms of its true and false Detection rate and is presented in table 4 . It is seen that the detection accuracy of the designed algorithm is better than existing. This is mainly due to the uses of motion vector in the Kalman filtering.

Table- IV: Comparison of Proposed Algorithm with Existing

\begin{tabular}{|c|c|c|}
\hline Authors & Techniques & $\begin{array}{c}\text { Maximum } \\
\text { Accuracy }\end{array}$ \\
\hline $\begin{array}{c}\text { Venkatesan et al. } \\
\text { [24] }\end{array}$ & $\begin{array}{c}\text { Automatic Traffic Monitoring in } \\
\text { Real-World Limited Bandwidth } \\
\text { Networks }\end{array}$ & $89.68 \%$ \\
\hline $\begin{array}{c}\text { Chih-Yang et al. } \\
\text { [25] }\end{array}$ & $\begin{array}{c}\text { Image Bit-Planes for Real-Time } \\
\text { Video Surveillance }\end{array}$ & $86.30 \%$ \\
\hline Yu and Fenfen [26] & Optical flow & $89.00 \%$ \\
\hline $\begin{array}{c}\text { Sridevi and } \\
\text { Meenakshi [27] }\end{array}$ & $\begin{array}{c}\text { Gaussian Mixture Model with } \\
\text { Morphological filter }\end{array}$ & $93.18 \%$ \\
\hline Proposed & $\begin{array}{c}\text { Modified Kalman Filter with } \\
\text { thresholding }\end{array}$ & $97.44 \%$ \\
\hline
\end{tabular}

\section{CONCLUSION}

In this paper, computer vision algorithm to efficiently compensate and detect the movement is proposed. The designed algorithm uses blob detection and modified Kalman filtering to compensate and detect the movements efficiently. Blob detection are used to improve the detection accuracy and the noise induced due to the changes in the light intensity and shadow effect are removed using thresholding technique. In order to consider the camera motion, the conventional Kalman filtering equations are replaced to compensate the motion caused by the movement of the camera which is used for capturing the video sequences. The experimental results shows that the developed algorithm can be used for compensating and detecting the movement efficiently. The comparison result with existing technique proves that the algorithm is efficient in estimating and detecting motion than existing.

\section{REFERENCES}

1. Isaac Cohen, G'erard Medioni "Detecting and Tracking Moving Objects for Video Surveillance" IEEE Proc. Computer Vision and Pattern Recognition Jun. 23-25, 1999. Fort Collins CO

2. Anand Paul and K. Bharanitharan, "Adaptive Edge Detection for Motion Estimation in H.264/AVC”. Seoul, South Korea, 2010.

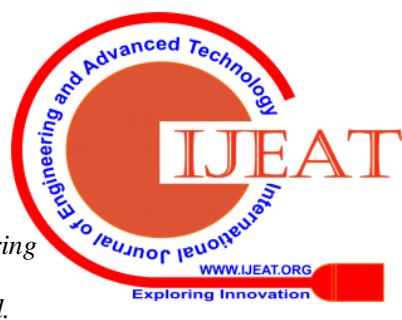


3. ZOU Xiao-chun, HE Ming-yi, ZHAO Xin-bo and FENG Yan, "A Robust Feature-Based Camera Motion Estimation Method" .International Conference on Innovative Computing and Communication and 2010 Asia-Pacific Conference on Information Technology and Ocean Engineering, China, 2010.

4. Pan Qing, Tian Nili and XuRuyi, "A Motion Estimation Algorithm Based on Inter-frame Differences Detection Model for Virtual Edge Detection", Seventh International Conference on Natural Computation, Wuhan, China, 2011.

5. Anthony Amankwah and Chris Aldrich, "Motion Estimation in Flotation Froth Images Based on Edge Detection and Mutual Information", IGARSS, Perth, Australia, 2012.

6. R Rukmani and Dr.M Jagadeeswari, "Error Detection and Correction Architecture for Motion Estimation in Video Coding SYSTEMS", international conference on computer communication and informatics, Coimbatore, INDIA, 2013

7. Yuming Wu, Siwen Deng and Wenwen Chen, "A Modified Octagon-Based Search Algorithm for Fast Block Motion Estimation", 2nd International Conference on Information Science and Contro Engineering, Loudi, P.R. China, 2015.

8. Muhammad Kamal Hossen and Sabrina Hoque Tuli, "A Surveillance System Based On Motion Detection And Motion Estimation Using Optical Flow", 5th International Conference on Informatics, Electronics and Vision (ICIEV), Chittagong, Bangladesh, 2016.

9. Raksha Umesh Bandana and Jinbeum Jang, Jieun Jo, Sangwoo Park and Joonki Paik, "Occlusion Detection Using Iterative Motion Estimation And Error Evaluation”, IEEE International Conference on Consumer Electronics-Asia (ICCE-Asia), Korea, 2016.

10. Yiming Wang, Qian Huang, Di Zhang and Yong Chen, "Digital Video Stabilization Based On Block Motion Estimation", International Conference on Computer Technology, Electronics and Communication (ICCTEC), Nanjing, China, 2017.

11. Rafael C. Gonzalez, Richard E. Woods and Steven L. Eddins, "Digital Image Processing Using MATLAB", Gatesmark Publishing, 2nd Edition, 2009.

12. J. Olivares, J. Hormigo, J. Villalba, I. Benavides and E.L. Zapata, "SAD computation based on online arithmetic for motion estimation", Microprocessors and Microsystems, Elsevier,Vol. 30 pp. 250-258, 2006.

13. Zhao-Yi Wei, Dah-Jye Lee, David Jilkand Robert Schoenberger, "Motion Projection for Floating Object Detection", International Symposium on Visual Computing, pp 152-161, 2007.

14. Gagandeep Kaur and SumeetKaur, " Moving Object Detection Using Graph's Axis Change Method", International Journal of Computer Technology \&Applications, Vol 3 (2), 701-704.

15. Trung Hieu Tran, Hyo-Moon Cho and Sang-Bock Cho, "Performance Enhancement of Sum of Absolute Difference (SAD) Computation in H.264/AVC Using Saturation Arithmetic", International Conference on Intelligent Computing, pp 396-404, 2009.

16. R. E. Kalman,, "A New Approach to Linear Filtering and Prediction Problems", Journal of Basic Engineering, Vol. 82, pp. 35-45, 1960.

17. D.V. Thombre; J H Nirmal; Das Lekha, " Human detection and tracking using image segmentation and Kalman filter", International Conference on Intelligent Agent \& Multi-Agent Systems, 2009.

18. Simon J. Julier, "The Scaled Unscented Transformation ", Proc. of the American Control Conference, pp. 555-559, May 2002.

19. Vasant Manohar, Padmanabhan Soundararajan, Harish Raju, Dmitry Goldgof, Rangachar Kasturi and John Garofolo, "Performance Evaluation of Object Detection and Tracking in Video", Asian Conference on Computer Vision, Springer, pp. 151-161, 2006.

20. Rafael C. Gonzalez, Richard E. Woods and Steven L. Eddins, "Digital Image Processing Using MATLAB", Gatesmark Publishing, $2^{\text {nd }}$ Edition, 2009.

21. Tao JIA, Nong-liang SUN, Mao-yong CAO, "Moving object detection based on blob analysis", IEEE International Conference on Automation and Logistics, pp. 322-325, China.

22. Swati and Gaurav Dixit, "Improved algorithm for blob detection in document images". $5^{\text {th }}$ International Conference - Confluence the Nex Generation Information Technology Summit, pp. 703-708, 2014, India.

23. Thanh Binh Nguyen and Sun Tae Chung, "An Improved Real-time Blob Detection for Visual Surveillance", 2nd International Congress on Image and Signal Processing, pp. 2-5, 2009, China.

24. K.G.S. Venkatesan, V. Khanaa, M.Sriram and S. Lekha, "An Advanced Moving Object Detection Algorithm for Automatic Traffic Monitoring in Real-World Limited Bandwidth Networks," IEEE International Journal of Innovative Research in Computer and Communication Engineering, vol.16, pp.2661-2668, March 2015.

25. L.Chih-Yang, J. Zhi-Yao, and L.Wei-Yang, "Image Bit-Planes Representation for Moving Object Detection in Real-Time Video
Surveillance, "IEEE International Conference on Consumer Electronics- Taiwan, pp.1-2, May 2016.

26. Z.Yu and W.Fenfen, "Improved optical flow algorithm of moving object Detection," IEEE International Conference on Instrumentation and Measurement, Computer, Communication and Control, pp.196-199, February 2016.

27. Sridevi N and M Meenakshi, "Efficient Moving Vehicle Detection Algorithm for Various Traffic Conditions," International Journal of Recent Technology ane Engineering, pp.1-2, July 2019.

28. Xi Chen, Xiao Wang and Jianhua Xuan, "Tracking Multiple Moving Objects Using Unscented Kalman Filtering Techniques", International Conference on Engineering and Applied Science, 2012.

\section{AUTHORS PROFILE}

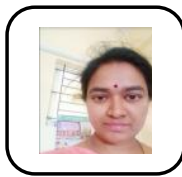

Sridevi $\mathbf{N}$ obtained her B. E. degree from UBDTCE Davangere and $\mathrm{M}$. Tech degree, from VTU, Karnataka in the year 1997 and 2007. Presently she is pursuing Ph.D, in Image and Video Processing at VTU, Karnataka. She is working as Associate Professor in Dr. Ambedkar Institute of Technology, Bangalore, Karnataka. She has 3 publications in International Journals and presented 25 papers at various International and National Conferences. Her areas of interests are Image and Video Processing, Process Automation and Robotics.

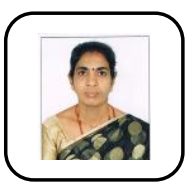

Dr. M. Meenakshi obtained her PhD from Department of Aerospace Engineering, Indian Institute of Science Bangalore, M.Tech from IIT Madras and B.E from SJCE Mysore. She is currently serving as Professor and Head of the Department of Electronics and Instrumentation Engineering, at Dr. Ambedkar Institute of Technology Bangalore. She was with Malnad college of Engineering Hassan as Faculty during 1987 - 2007. She has around 115 research publications in her credit, which includes International Journals, International Conferences, National Conference, Technical Books, and Book Chapters. 